\title{
Lateral metallic devices made by a multiangle shadow evaporation technique
}

\author{
Marius V. Costache and Germàn Bridoux \\ Catalan Institute of Nanotechnology (ICN) Bellaterra, Barcelona E-08193, Spain \\ Ingmar Neumann \\ Catalan Institute of Nanotechnology (ICN), and Physics Department, Universitat Autònoma de Barcelona. \\ Bellaterra, Barcelona E-08193, Spain
}

Sergio O. Valenzuela

Institució Catalana de Recerca i Estudis Avançats (ICREA), Catalan Institute of Nanotechnology (ICN), and Physics Department, Universitat Autònoma de Barcelona. Bellaterra, Barcelona E-08193, Spain

(Received 15 February 2012; accepted 13 May 2012; published 30 May 2012)

\begin{abstract}
The authors report the fabrication of lateral metallic structures with multiple materials using specifically designed resist masks and multiangle shadow evaporation. The whole fabrication process is carried out without breaking vacuum, which avoids contamination and allows for highly controlled interface properties between metals deposited sequentially. The authors incorporate the thickness of the mask as a design parameter, which allows one to introduce controlled variations between multiple contacts in the same device. Using a suspended mask, it is demonstrated the fabrication of asymmetric single electron transistors with tunnel junctions with different resistances. Using a nonsuspended mask, it is illustrated the fabrication of an extended structure (a thermopile), which consists of tenths of ferromagnetic wires with a nominal width of $30 \mathrm{~nm}$ connected electrically in series using a nonmagnetic metal. (c) 2012 American Vacuum Society. [http://dx.doi.org/10.1116/1.4722982]
\end{abstract}

\section{INTRODUCTION}

The development of novel (metallic) devices in recent years has been closely related to new methods and techniques to fabricate them. In situ fabrication methods are gathering increasing attention due to the use of multiple metals in the same device and the stringent need to control the interfaces between them. For example, submicron metal/ $\mathrm{AlO}_{\mathrm{x}} /$ metal tunnel junctions have multiple applications ${ }^{1-5}$ but the fabrication of reproducible junctions and a detailed study of their stability can only be carried out in a controlled way by minimizing contamination. This can be achieved using in situ bilayer double angle evaporation methods. ${ }^{1,6,7}$ There, a suspended mask is defined to fabricate the device by sequentially evaporating the materials involved from different angles without breaking vacuum in the whole process (Fig. 1). In this way, one can create very clean interfaces or well-controlled tunnel junctions. Because of such capabilities, methods involving suspended masks have become very popular for superconducting qubits, ${ }^{2}$ spintronics, ${ }^{4,5}$ and in single charge electronics. ${ }^{1}$ In spintronics, multiterminal lateral devices have been used to determine spin relaxation lengths, ${ }^{4-11}$ to study the spin Hall effect, ${ }^{12,13}$ or to demonstrate spin ratchet ${ }^{14}$ and magnon drag ${ }^{15}$ effects.

Standard in situ methods using a resist bilayer and a double-angle evaporation technique have enabled ${ }^{6,7}$ significant progress in sample fabrication and are an established technique in mesoscopic physics. However, they are restricted to only two materials and to interfaces with nominally the same properties, even in a device with multiple contacts. A suspended mask also restricts the separation between the device features, limits the achieved resolution, and it is not suitable for extended complex structures. In this article, we describe in detail the implementation of multiangle evaporation methods that incorporate the height of the top resist as a variable for mask design. This is a novel strategy that allows us to overcome the aforementioned limitations of the standard shadow evaporation technique. The top resist can be used to selectively eliminate specific features to be projected on the substrate at specific evaporation angles. By allowing evaporation from any direction, as opposed to only two directions, ${ }^{1,6,7}$ extended complex structures with more than two materials can easily be implemented. Following this description, we describe in detail two examples for the enhanced shadow evaporation method. First, we demonstrate a three-angle evaporation technique to create single electron transistors for which the two tunnel junctions can be controlled independently. Second, we describe the fabrication of a thermopile with ferromagnetic components comprising more than 40 wires with a nominal width of $30 \mathrm{~nm}$ connected electrically in series. The fabrication of these devices requires a careful design of the mask features, the feature distribution, and the mask thickness. After fabrication, we present the transport characteristics of the final devices, which show a high level of control of the interfaces. ${ }^{14,15}$

\section{DEVICE FABRICATION}

The main mask elements to create a contact between two materials are represented in Figs. 1 and 2. In Fig. 1(a), we show the standard two-angle evaporation scheme. Here the features of the basic mask involve two regions separated by a small suspended bridge. Sequential metal evaporation between two properly chosen angles results in a contact between the shadows of the two features in their overlapping region, as marked in Fig. 1(b). Normally, the sample is rotated about an axis within the sample plane and thus the evaporation directions form a plane perpendicular to the mask. 
(a)

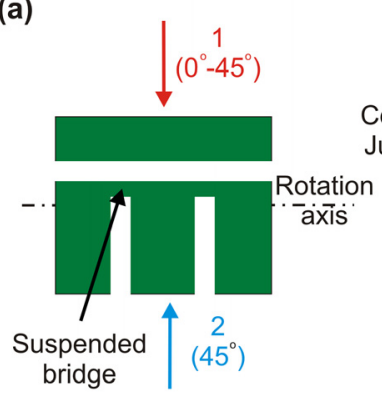

(b)

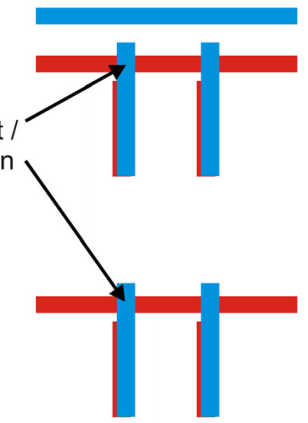

(d)

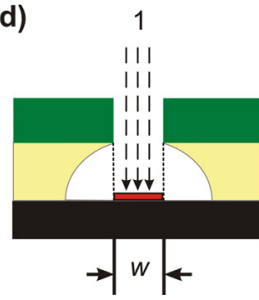

PMMA

MMA

$\mathrm{Si} / \mathrm{SiO}_{2}$

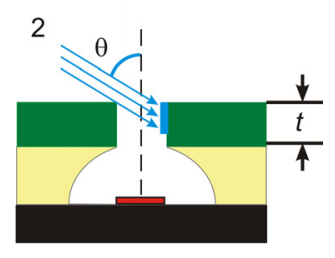

FIG. 1. (Color online) Suspended mask. (a) Mask features seen from above. A contact between two materials is achieved by using a mask with a small suspended bridge. (b) Material deposition resulting from double angle evaporation through a conventional mask. (c) Material deposition using a thick mask. The features (nearly) parallel to the sample rotation axis do not project a shadow in the substrate and the evaporated material deposits on the mask lateral walls (d) and is later removed by lift-off.

This technique has been used in the fabrication of single electron transistors and superconducting qubits. ${ }^{1}$ It has, however, important limitations. For instance, all of the contacts are made simultaneously and therefore have the same characteristics. Additionally, the multiple shadows of the mask features restrict the complexity of the design or generate unwanted contacts or tunnel junctions. ${ }^{16}$ However, one can eliminate some of the unwanted contacts by controlling the height of the top resist [Figs. 1(c) and 1(d)].

Figure 1(d) shows that material deposition straight through the mask, i.e., perpendicular to the substrate, results in the projection of all the mask features onto the substrate (left). When the material is deposited at an angle $\theta$ [Fig. 1(d), right], some of the features with a small width, $w$, perpendicular to the axis of rotation do not have a projection onto the surface. For this to be possible, the rotation axis, the angle, $\theta$, the thickness of the top resist, $t$, and the feature size, $w$, have to be properly designed, and should fulfill the simple inequality: $t>w / \tan \theta$. Note that the axis of rotation does not need to be exactly parallel to the length of the mask feature. If there is a misalignment $\phi$ between the axis of rotation and the width of the feature, the relation becomes: $t>w /(\cos \phi \tan \theta)$. Such an idea was implemented in Refs. 9 and 12 and is used in its full extent in a second shadow evaporation method as shown in Fig. 2. In this case, the sample rotates about an axis perpendicular to the sample plane and the evaporation directions do not form a plane perpendicular to the mask. ${ }^{15,17-19}$ In the simplest case, the material deposition is done from a constant angle from the substrate normal. In Fig. 2, this angle is set to be $45^{\circ}$. However, the combination of the methods in Figs. 1(d) and 2 can be utilized for increasing the complexity of the structures.

(a)

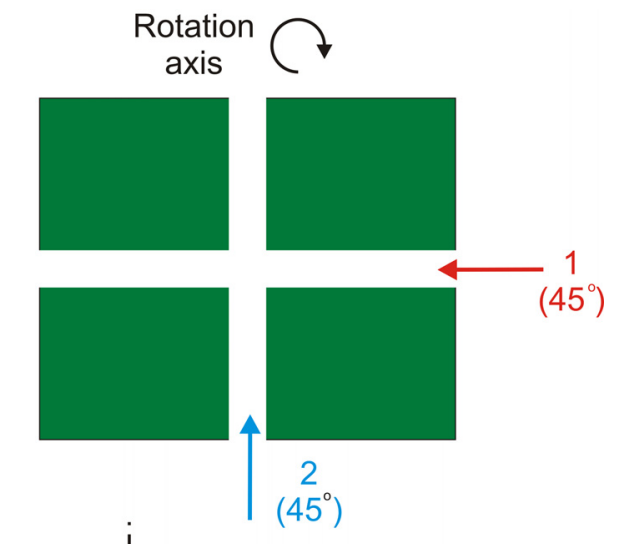

(b)

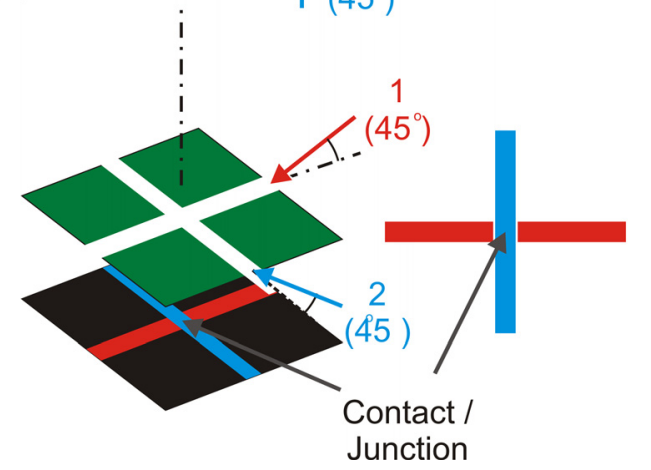

FIG. 2. (Color online) Nonsuspended mask. (a) Mask features seen from above. A contact between two materials is achieved by using a mask with a cross shape. (b) The sample rotates about an axis perpendicular to the sample plane and the evaporation directions do not form a plane perpendicular to the mask.

Below, we describe two examples that illustrate the implementation of the methods introduced above.

\section{A. Fabrication of contacts with variable characteristics using multiple materials}

Here we describe the fabrication of asymmetric single electron transistors. The fabrication of this device involves a three angle evaporation method and the use of a top resist with large thickness to prevent small features from creating unwanted metal contacts. This device was designed to demonstrate a spin ratchet concept, which achieves directed spin transport in the presence of a signal or perturbation without a control bias. The device is a single electron transistor (SET) with a small superconducting island and normal metal leads, where the tunnel junctions contacting the SET have to be controlled independently. ${ }^{14}$

The SETs consist of a small-volume $(6 \mathrm{~nm}$ thick $\times 40 \mathrm{~nm}$ wide $\times 250 \mathrm{~nm}$ long) aluminum ( $\mathrm{Al}$ ) island (S) connected to two electrodes, N(l) and N(r). Figure 3 shows the main elements for their fabrication, which involve electron-beam lithography and multiangle shadow evaporation to produce tunnel barriers in situ. The suspended shadow mask (Fig. 3) is first created on a highly- doped $\mathrm{Si}(100)$ wafer with thermally grown oxide. To this end, we use a methyl methacrylate (MMA)/poly(methyl methacrylate) (PMMA) bilayer in combination with selective electron- beam exposure. The base resist (MMA) has a sensitivity that is about 5 times larger than the top resist (PMMA), which allows us to generate a 
(a)

(b)
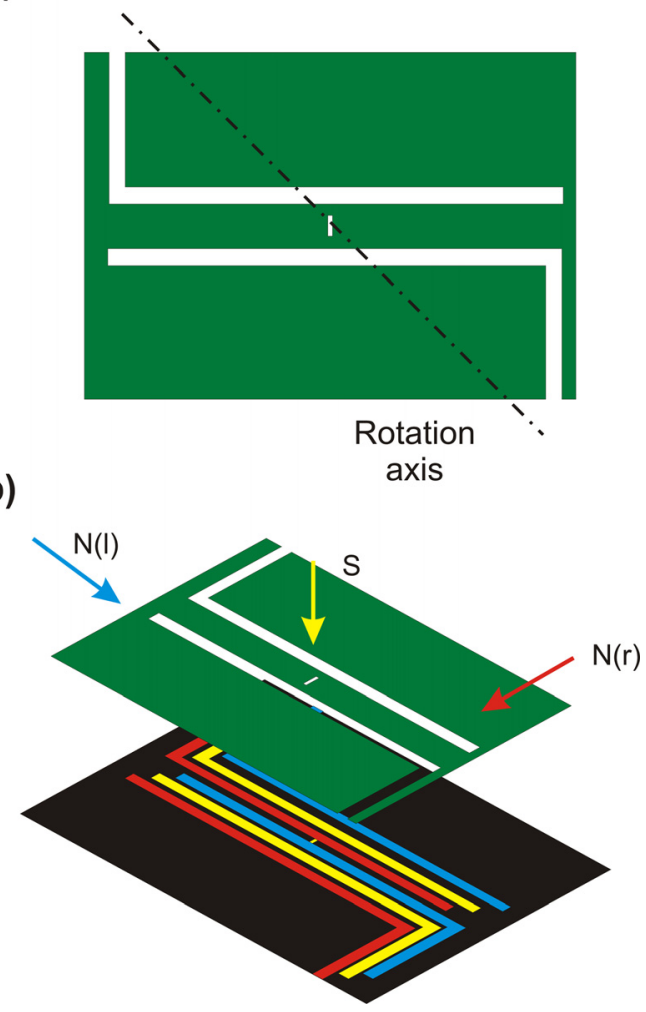

FIG. 3. (Color online) Asymmetric single electron transistor. (a) Design of the suspended MMA/PMMA mask for shadow evaporation. The dashed line represents the rotation axis for shadow evaporation. (b) The device is fabricated by three sequential depositions as indicated by the arrows. Such a process results in a threefold projection of the mask.

controlled undercut by exposing the bilayer with a dose that is sufficient to expose the MMA layer, but insufficient to expose the PMMA layer. The exposed bilayer is developed in an isopropanol/methyl-isobutyl-ketone (IPA:MIBK; 3:1) solution and placed in a high-vacuum electron-beam evaporator (base pressure $\leq 10^{-8}$ Torr). This fabrication not only allows to control the tunnel junction properties independently but also to deposit three different materials sequentially.

The material evaporation sequence is shown in Figs. 3(a) and 3(b). First, we evaporate Al perpendicular to the substrate (yellow), which creates the island. Next, $\mathrm{Al}$ is oxidized in pure oxygen (100-150 $\mathrm{mTorr}$ for $40 \mathrm{~min}$ ) to generate insulating $\mathrm{Al}_{2} \mathrm{O}_{3}$ barriers. After the vacuum is recovered, the two electrodes, N(l) (blue) and N(r) (red), are sequentially deposited under angles of $50^{\circ}$ relative to the substrate normal, where the substrate is tilted in the opposite direction for $\mathrm{N}(\mathrm{l})$ and N(r) [Fig. 3(b)]. The sequential deposition leads to different tunneling resistances $R_{l}$ and $R_{r}$; the difference between $R_{l}$ and $R_{r}$ can be enhanced by an additional oxidation step in between each lead deposition. The three-angle metal deposition results in a threefold projection of all the mask features with a spatial shift, except for the island, which is deposited only once. The axis of rotation [indicated by a dashed line in Fig. 3(a)] is selected such that the island feature at $50^{\circ}$ tilting projects onto the sidewall of the top PMMA resist and later on the deposited material is removed by lift-off. In this case, the top resist has to be thicker than the projection of the (a)

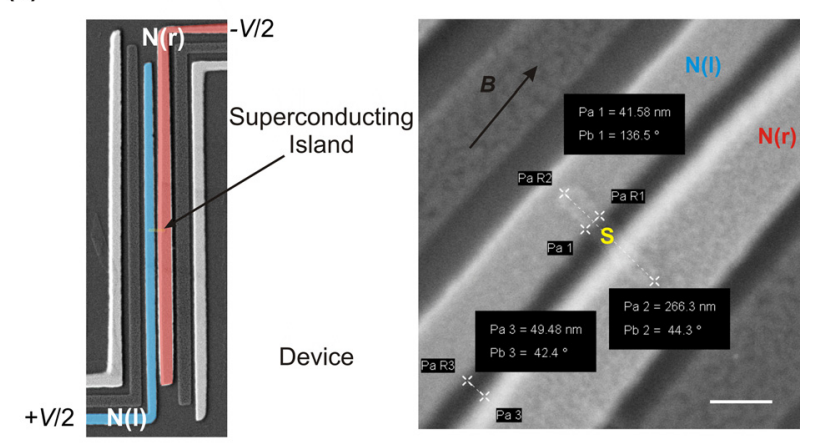

(b)

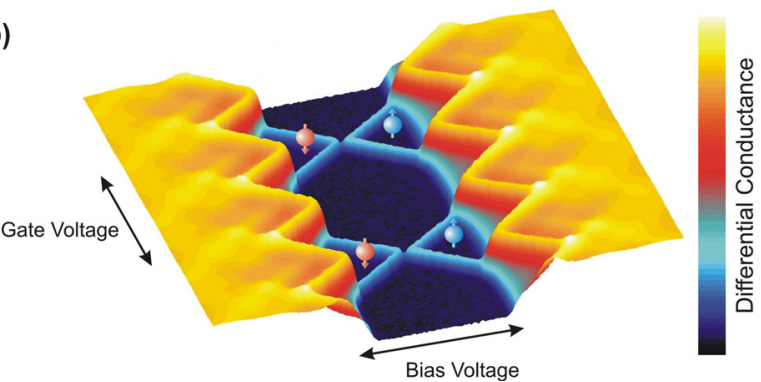

FIG. 4. (Color online) (a) SEM image of a finished device. The bar is $100 \mathrm{~nm}$. (b) Experimental conductance $\mathrm{d} I / \mathrm{d} V$ as a function of dc voltage $V$ across the SET and gate voltage $V_{g}$ in a logarithmic scale to highlight the SET subgap transport at a temperature of $25 \mathrm{mK}$ and at a magnetic field $B=1.5 \mathrm{~T}$, where the conditions for spin pumping/spin ratchet are fulfilled (see Ref. 15). The $\mathrm{d} I / \mathrm{d} V$ amplitude is represented by a color scale from black (zero) to yellow $(15 \mu \mathrm{S})$.

island in a perpendicular plane [see Fig. 1(d)]. For our island that has less than $50 \mathrm{~nm}$ in width, a $100 \mathrm{~nm}$ top layer of PMMA suffices. A detail of a finished sample [Fig. 4(a)] shows the lack of secondary islands and contact leads that are very close to each other $(\sim 50 \mathrm{~nm})$, which is not straightforward to achieve with standard masks [Fig. 1(b)].

As an example of the quality of the SET, we use differential conductance measurements $\mathrm{d} I / \mathrm{d} V$ at above-gap voltages which allow us to determine the device parameters [Fig. 4(b)], including the junctions' capacitances $C_{l}$ and $C_{r}$, the gate capacitance $C_{g}$, and the superconducting gap, $\Delta$. Measurements were performed in a dilution refrigerator at $25 \mathrm{mK}$ with a true four-point ac/dc data acquisition technique. A dc voltage and a small superimposed ac sine voltage $(20 \mu \mathrm{V})$ are applied to the SET. Both the ac current component through the SET and the ac voltage across the normal leads are acquired using standard lock-in techniques. From the $\mathrm{d} I / \mathrm{d} V$ thresholds in Fig. 4(c) the following parameters are obtained: $\Delta=303 \mu \mathrm{eV}$, $C_{l} \sim C_{r} \approx 235 \mathrm{aF}, C_{g} \approx 1.4 \mathrm{aF}, C_{\sum}=C_{l}+C_{r}+C_{g} \approx 470 \mathrm{aF}$, and $E_{c}=e^{2} / 2 C_{\sum} \approx 170 \mu \mathrm{eV}$. Measurements in Ref. $14 \mathrm{dem}-$ onstrate that the desired asymmetry in the junctions was achieved.

\section{B. Extended structures}

Here we describe the fabrication of a thermopile. The mask for this device (Fig. 5) exemplifies the case described in Fig. 2. The thermopile was designed for the detection of the magnon drag effect. ${ }^{15}$ Magnon drag should be observed 
(a)

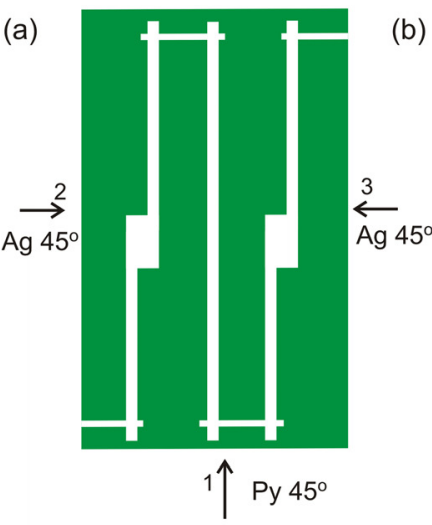

(c)

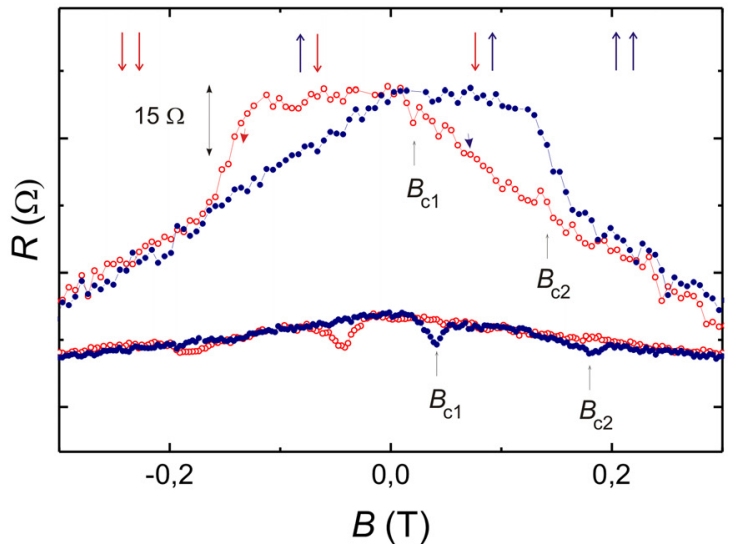

FIG. 5. (Color online) Fabrication of a thermopile and transport characterization. (a) A partial view of the design of the shadow mask made with e-beam lithography using a PMMA/MMA bilayer on a $\mathrm{SiO}_{2} / \mathrm{Si}$ substrate. The metal deposition sequence is shown. (b) Scanning electron microscope image of a finished device. The central part of the device is formed by 20 Py wire-pairs connected at both ends with $\mathrm{Ag}$ wires. The dc voltage generated between the end electrodes is measured as a function of a temperature gradient created with an on-chip Pt heater (bright vertical strip) in close proximity to the Py wires. (c) MR at $295 \mathrm{~K}$ (top curves) and at room temperature (bottom curves). The $B$ swept direction is from $B>0$ to $B<0$ (red) and from $B<0$ to $B>0$ (blue). The arrows indicate the parallel/antiparallel magnetization configuration of Py wires. The measurements were displaced vertically for clarity.

in a ferromagnet wire under a temperature gradient due to electron-magnon scattering. Our devices consist of a large number of pairs of permalloy (NiFe) wires $(30 \mathrm{~nm}$ wide, $20 \mathrm{~nm}$ thick, and $5 \mu \mathrm{m}$ long) connected in a zigzag configuration by short silver ( $\mathrm{Ag}$ ) wires ( $40 \mathrm{~nm}$ thick). Each NiFe wire in a pair is identical to the other except for their geometry that results in different coercive fields that allow us to control the relative orientation of their magnetizations. The device resembles a thermopile formed by the large number of $\mathrm{NiFe}$ wires placed between a hot and a cold source and connected thermally in parallel and electrically in series.

A scanning electron microscope image (SEM) of a typical device is shown in Fig. 5(b). The main elements for the device fabrication involve two electron-beam lithography steps and angle shadow evaporation [Fig. 5(a)]. A heater consisting of a $50 \mathrm{~nm}$ thin film of Pt with a $5 \mathrm{~nm}$ Ti adhesion layer is defined in the first lithographic step. Next, a shadow mask is fabricated as described below and the thermopile is formed by depositing, sequentially, the $\mathrm{NiFe}$ and the $\mathrm{Ag}$ wires, where the latter connect the $\mathrm{NiFe}$ wires electrically in series. The shadow evaporation allows for material deposition without breaking the vacuum, therefore producing clean contacts between $\mathrm{NiFe}$ and $\mathrm{Ag}$. The use of in situ deposition is crucial due to the number of contacts involved. An alternative way to fabricate such devices consists of multiple electron beam lithography steps and ion cleaning between successive metal evaporations. However, such a cleaning process is rather low yield, which, combined with the large number of contacts, would reduce the yield of working devices (because the wires are in series and if one of these contacts fails, then the device will fail).

The shadow mask [Fig. 5(a)] is fabricated on a highlydoped $\mathrm{Si}(100)$ wafer with thermally grown oxide. It uses the concept sketched in Fig. 2. As before, we start with the MMA/ PMMA bilayer in combination with selective electron-beam exposure. The exposed bilayer is again developed in an IPA:MIBK (3:1) solution and placed in a high-vacuum electronbeam evaporator (base pressure about $10^{-8}$ Torr). In this case, the undercut does not need to be as large as in the previous example and it is only used to improve lift-off. The material evaporation sequence is shown in Fig. 5(a). First, we evaporate $\mathrm{NiFe}$ under an angle of $45^{\circ}$ relative to the substrate normal, which creates the NiFe wires. Next, the substrate is rotated $90^{\circ}$ around an axis normal to the substrate and sequentially tilted in opposite directions $\left( \pm 45^{\circ}\right)$ to create continuous electrodes by $\mathrm{Ag}$ evaporation. With the $45^{\circ}$ tilt only the mask features in the deposition plane that contains the substrate normal and the evaporation source project onto the substrate. The rest of the mask features result in material deposition onto the sidewall of the top PMMA resist, which later on is removed by lift-off. For this purpose, the height of the top resist has to exceed the width of the larger feature, which in this case is about $200 \mathrm{~nm}$ (note that the angle is $45^{\circ}$ ). Such height can be readily obtained with commercial PMMA with the right molecular weight. For example, PMMA with a molecular weight of $950 \mathrm{~K}$ diluted at $3 \%$ in chlorobenzene spun onto a substrate at $4000 \mathrm{rpm}$ would result in a film of $300 \mathrm{~nm}$. The features in the PMMA mask must have a large aspect ratio. For them to be well- defined, it is therefore necessary to use an electron beam lithography system with large accelerating voltages $(\geq 30 \mathrm{keV})$.

Electrical characterization of the finished device demonstrates excellent electrical contacts between the large number of wires [Fig. 5(c)]. We used magnetoresistance (MR) measurements at 4.2 and $295 \mathrm{~K}$, with a magnetic field applied along the wires. At $4.2 \mathrm{~K}$ the $\mathrm{MR}$ is readily explained by anisotropic magnetoresistance. The resistance of the thermopile shows weak field dependence except about the two narrow minima, which correspond to the switching fields $B_{c 1}, B_{c 2}$ of the two wires geometries. At $295 \mathrm{~K}$ the magnon contribution to MR clearly dominates, the resistance shows a nonsaturating linear decrease with field which is due to a softening of the magnon-electron scattering, and a decrease of magnon population with increasing the field. ${ }^{15,20,21}$ Such small variations of the resistance can only be observed in samples with clean low- 
resistance contacts. If contacts dominate the overall resistance the relative change due to magnetoresistance effects could easily be masked by noise.

\section{SUMMARY AND CONCLUSIONS}

We described in detail the implementation of multiangle evaporation methods that incorporate the height of the top resist as a variable for mask design, which allowed us to introduce controlled variations between multiple contacts in the same device and to fabricate complex structures, while avoiding contamination. Following this description, we provided two examples for the enhanced shadow evaporation method. The examples presented in this work are closely related to spintronics. However, we expect that the involved fabrication methods will also prove relevant in other fields, in particular for complex superconducting circuits, including multiple-qubit systems. First, we demonstrated a technique to create single electron transistors for which the island can be very small and the two tunnel junctions can be controlled independently. In this case, the evaporation of material onto the mask walls eliminated the deposition of multiple shadows of the transistor island, which enabled us to contact the island using three-angle evaporation over a suspended mask. The sequential deposition of the contacts and multiple oxidation steps allow the independent control of the tunnel barrier resistances and electrodes that can be very close. Second, we demonstrated the fabrication of a thermopile with ferromagnetic components comprising more than 40 wires with a nominal width of $30 \mathrm{~nm}$ that were connected electrically in series. In this case, we used a nonsuspended mask which presents the advantage of being robust for complex extended features without affecting the attained resolution.

\section{ACKNOWLEDGMENTS}

This research was supported by the Spanish Ministerio de Ciencia e Innovación, MICINN (MAT2010-18065 and FIS2009-06671-E) and by the European Community's Seventh Framework Programme (FP7/2007-2013) under grant agreement NANOFUNCTION No. 257375.

${ }^{1}$ T. A. Fulton and G. J. Dolan, Phys. Rev. Lett. 59, 109 (1987).

${ }^{2}$ C. H. van der Wal, A. C. J. ter Haar, F. K. Wilhelm, R. N. Schouten, C. J. P. M. Harmans, T. P. Orlando, Seth Lloyd, and J. E. Mooij, Science 290, 773 (2000).

${ }^{3}$ W. D. Oliver and S. O. Valenzuela, Quantum Inf. Process. 8, 261 (2009).

${ }^{4}$ F. J. Jedema, H. B. Heersche, A. T. Filip, J. J. A. Baselmans, and B. J. van Wees, Nature 416, 713 (2002).

${ }^{5}$ S. O. Valenzuela, Int. J. Mod. Phys. B 23, 2413 (2009).

${ }^{6}$ G. J. Dolan, Appl. Phys. Lett. 31, 337 (1977).

${ }^{7}$ I. M. Pop, T. Fournier, T. Crozes, F. Lecocq, I. Matei, B. Pannetier,

O. Buisson, and W. Guichard, J. Vac. Sci. Technol. B 30, 010607 (2012).

${ }^{8}$ F. J. Jedema, M. V. Costache, H. B. Heersche, J. J. A. Baselmans, and B. J. van Wees, Appl. Phys. Lett. 81, 5162 (2002).

${ }^{9}$ S. O. Valenzuela and M. Tinkham, Appl. Phys. Lett. 85, 5914 (2004).

${ }^{10}$ Y. Ji, A. Hoffmann, J. E. Pearson, and S. D. Bader, Appl. Phys. Lett. 88, 052509 (2006).

${ }^{11}$ T. Kimura and Y. Otani, Phys. Rev. Lett. 99, 196604 (2007).

${ }^{12}$ S. O. Valenzuela and M. Tinkham, J. Appl. Phys. 101, 09B103 (2007).

${ }^{13}$ L. Vila, T. Kimura, and Y. Otani, Phys. Rev. Lett. 99, 226604 (2007).

${ }^{14}$ M. V. Costache and S. O. Valenzuela, Science 330, 1645 (2010).

${ }^{15}$ M. V. Costache, G. Bridoux, I. Neumann, and S. O. Valenzuela, Nature Mater. 11, 199 (2012).

${ }^{16}$ I. Chiorescu, Y. Nakamura, C. J. P. M. Harmans, and J. E. Mooij, Science 299, 1869 (2003).

${ }^{17}$ T. Yang, T. Kimura, and Y. Otani, Nature Phys. 4, 851 (2008).

${ }^{18}$ G. Bridoux, M. V. Costache, J. Van de Vondel, I. Neumann, and S. O. Valenzuela, Appl. Phys. Lett. 99, 102107 (2011).

${ }^{19}$ P. Łączkowski et al., Appl. Phys. Express 4, 063007 (2011).

${ }^{20}$ A. P. Mihai, J. P. Attané, A. Marty, P. Warin, and Y. Samson, Phys. Rev. B 77, 060401(R) (2008).

${ }^{21}$ V. D. Nguyen L. Vila, P. Laczkowski, A. Marty, T. Faivre, and J. P. Attané, Phys. Rev. Lett. 107, 136605 (2011). 